\title{
A Text Recognizing Device For Visually Impaired
}

\author{
G.Tejaswani ${ }^{1}$, Afroz.B ${ }^{2}$, Sunitha. $S^{3}$ \\ 1 G.Tejaswani (UG), $8^{\text {th }}$ SEM Department of Computer Science \& Engineering, \\ Rao Bahadhur Y. Mahabaleswarappa Engineering \\ Collage, Ballari, Karnataka, India. \\ 2 Afroz.B (UG), $8^{\text {th }}$ SEM Department of Computer Science \& Engineering, \\ Rao Bahadhur Y. Mahabaleswarappa Engineering \\ Collage, Ballari, Karnataka, India. \\ 3 Assistant.prof Sunitha.S, Department of Computer Science \& Engineering, \\ Rao Bahadhur Y. Mahabaleswarappa Engineering \\ Collage, Ballari, Karnataka, India.
}

\begin{abstract}
:
In the present world visually impaired people use Braille lipi language to read the books, but those books are available only in particular places like library etc. It has become very difficult for the visually impaired peoples to come out into the real world. Braille cannot be used in every place like in the mobiles, supermarket, and news papers. Due to this it has become difficult for the visually impaired people to read the text. To overcome this difficulties faced by the visually impaired, finger reader with speech assistance is used to read any printed text from the mobiles, news papers etc. we are using the assistive technology in this device to provide the assistance for the visually impaired people.
\end{abstract}

Keywords: assistance technology, text reading.

\section{Introduction}

Accessing text has become very difficult for the visually impaired (VI) people. As per the survey the available technology such as screen reader, desktop scanner, smart phone application and eBook reader are commonly underutilized due to slow processing speeds or poor accuracy. To overcome these problems faced by the visually impaired people we can make use of the wearable device access the text easily and accurately. We can use this device just by wearing this to our pointer finger and pointing to the text which we want to read. By making use of this device visually impaired people can easily read the news paper, the text messages from the mobile without anybody help.

\section{Purpose}

The main purpose of this paper is to make visually impaired people to read the text content without anybody help. This device can be used at any time, in any place and it can read the content from any device or from the books.

\section{Literature survey}

\subsection{Eye Ring: An Eye on a Finger}

Author: Suranga Nanayakkara, Roy Shilkrot, Patricia Maes

Finger-worn devices are a greatly underutilized form of interaction with the surrounding world. By putting a camera on a finger we show that many visual analysis applications, for visually impaired people as well as the sighted, prove seamless and easy. We present finger reader, a ring mounted camera, to enable applications such as identifying currency and navigating, as well as helping sighted people to tour an unknown city or intuitively translate signage. The ring apparatus is autonomous however our system also includes a mobile phone or computation device to which it connects wirelessly, and an earpiece to receive information. Finally, we will discuss how different finger worn sensors may be extended and applied to other domains. 
3.2 Finger-mounted reading device for the blind Author: Suranga Nanayakkara

The paper also reports the results of a usability study conducted with vision-impaired volunteers, in which the researchers tested several variations of their device. One included two haptic motors, one on top of the finger and the other beneath it. The vibration of the motors indicated whether the subject should raise or lower the tracking finger.

\subsection{Finger Reader: A Wearable Device to Explore Printed Text on the Go}

Authors: Roy Shilkrot, Jochen Huber1, Meng Ee Wong, Pattie Maes, Suranga Nanayakkara

Accessing printed text in a mobile context is a major challenge for the blind. A preliminary study with blind people reveals numerous difficulties with existing state-of-the-art technologies including problems with alignment, focus, accuracy, mobility and efficiency. In this paper, we present a fingerworn device, Finger Reader that assists blind users with reading printed text on the go. We introduce a novel computer vision algorithm for local-sequential text scanning that enables reading single lines, blocks of text or skimming the text with complementary, multimodal feedback. This system is implemented in a small finger-worn form factor that enables a more manageable eyes-free operation with trivial setup. We offer findings from three studies performed to determine the usability of the Finger Reader.

\section{Early days}

In the early days visually impaired people use to read the books which were written in Braille lipi language, to read those books they should have the knowledge about Braille language how it written. They have take class about the Braille language to read that books.

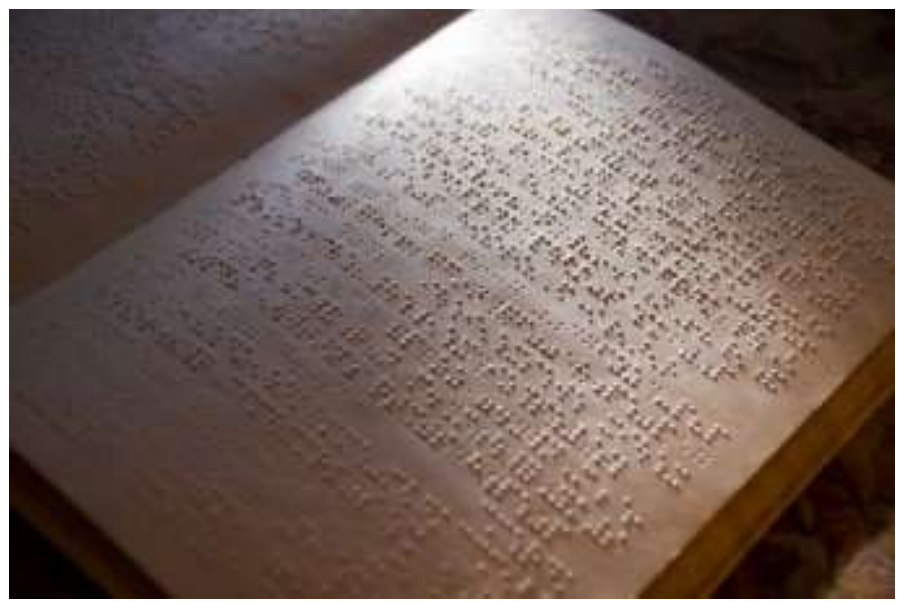

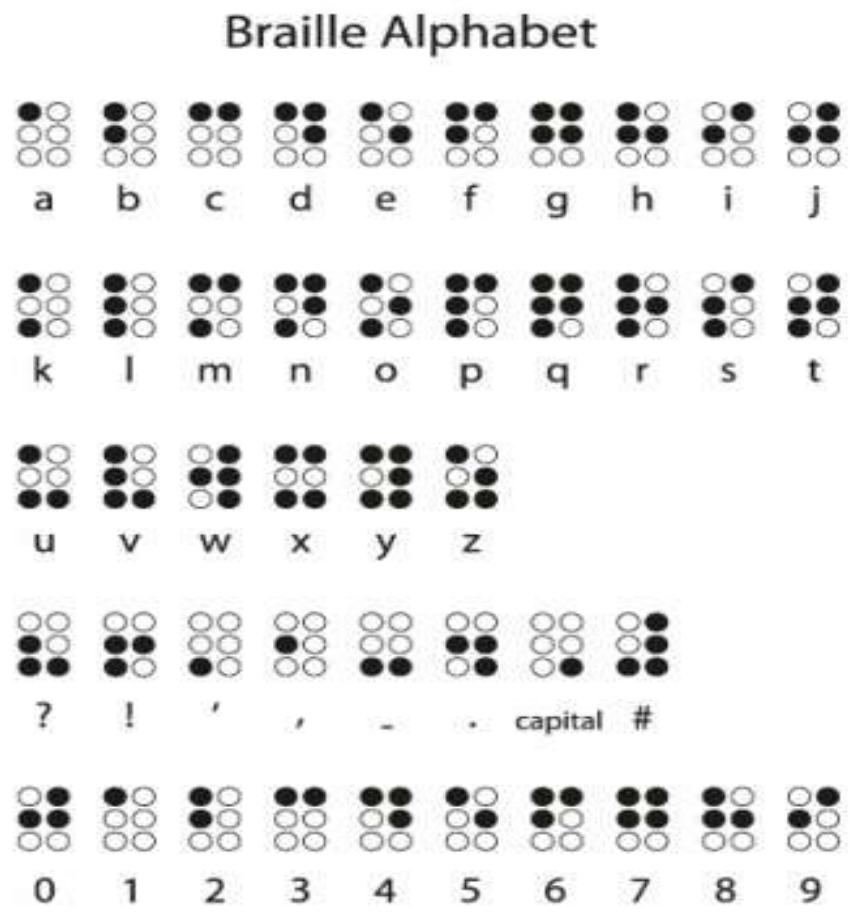

Fig 2: Braille Alphabets

\section{Working of the finger reader}

This is the device which was initially developed for visually impaired however this work features novel hardware and software that includes hepatic response, video-processing algorithms and different output modalities.

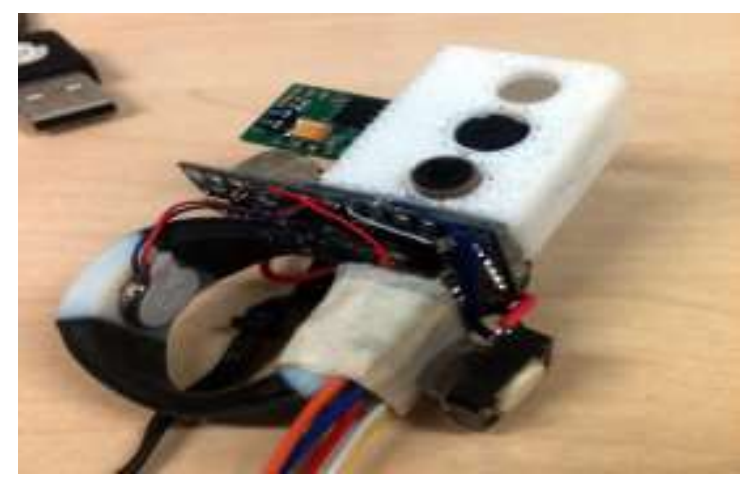

Fig 3: Multi-material prototype

To overcome these problems we have prepared this device for the visually impaired people. To make use of this device we have to just wear this device to our index finger and point to the text which we want to read. A camera is fixed in that device, which scans the words to which we are pointing to and gives the voice result.

Fig 1: Braille lipi 


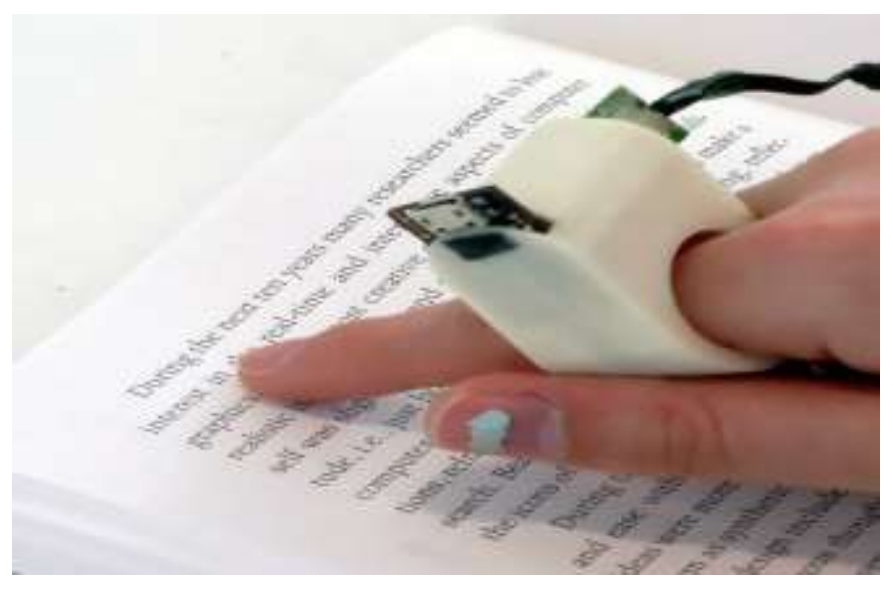

Fig 4: device used to recognize the text and read text for the user.

It is connected to the computer system or to the mobile while reading the text. It detects each and every word which is scanned by the camera and gives the voice output to the user, so that the user can easily understand the printed text from the books, news paper, mobile etc. The device performs this operation through complicated image processing.
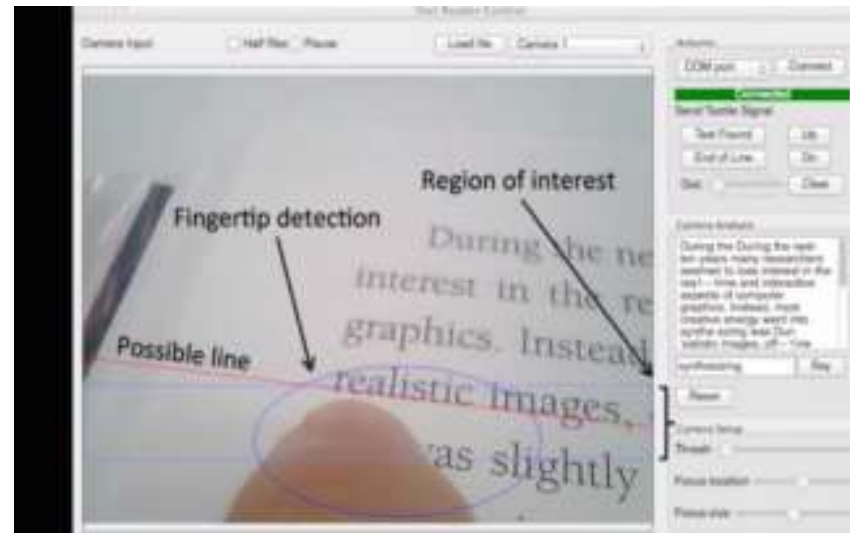

Fig 5: Recognizing the text through image processing.

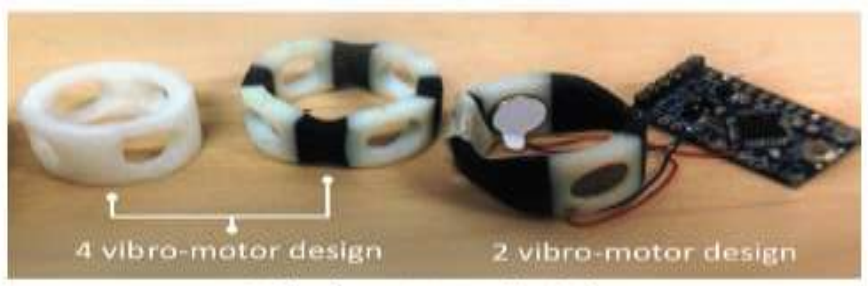

(a) Early prototypes Evolution
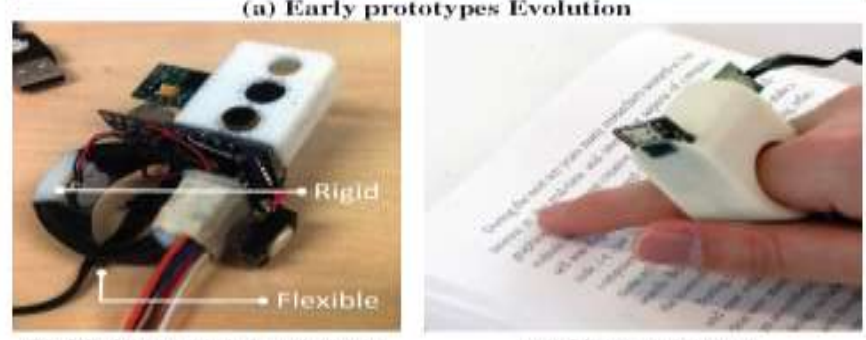

(c) New prototype (b) Multi-material prototype

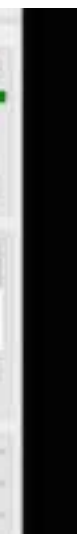

While accessing the text in an order if the user misses the line while reading, the device vibrates to indicate that user is reading wrong line. It also indicates the end of the line by vibrating and by giving the voice output. Two vibration motors are embedded on the top and bottom of the ring to provide haptic feedback on which direction the user should move the camera via distinctive signals. The dual material design provides flexibility to the ring's fit as well as helps dampen the vibrations and reduce confusion for the user.

To make the hardware work according to the user required, we develop software which includes the text extraction algorithm. We have to provide the input to the text extraction algorithm, a closed up printed text image is given as the input to that algorithm.

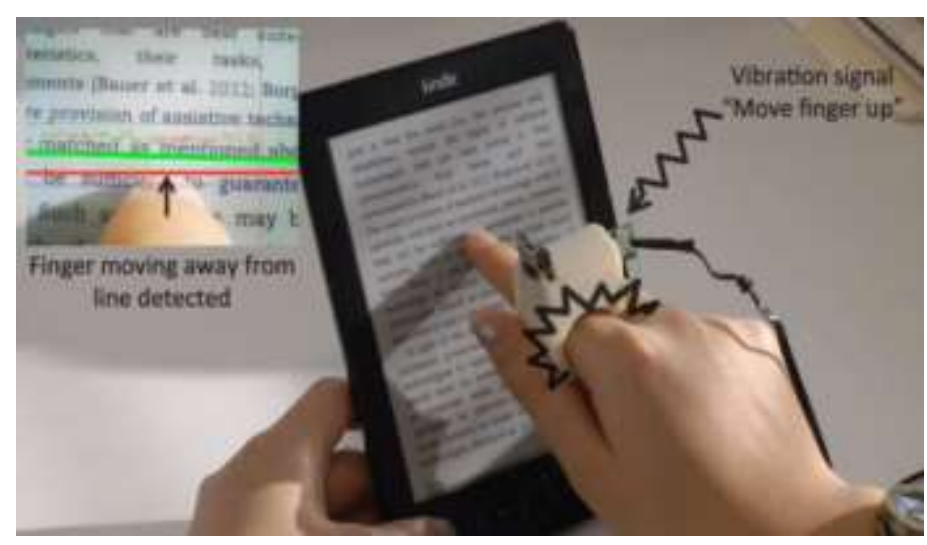

Fig 7: The Finger Reader warns the readers when they move away from the line.

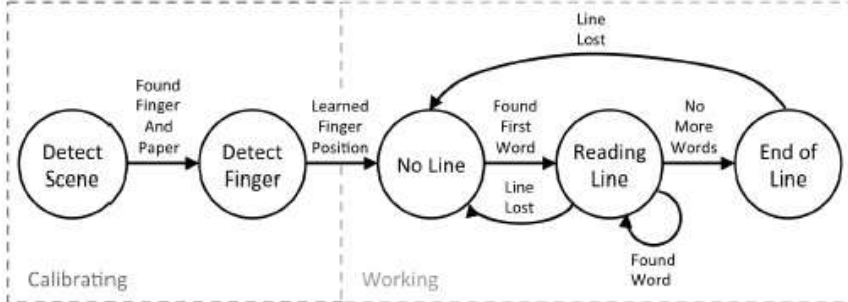

Figure 3: Sequential text reading algorithm state machine.

Fig 8:Sequence diagram of finger reader

It is the sequence diagram which shows the working of the finger reader. Initially after wearing the device to finger it detects the paper this is know as detect scene. Then it detects the finger this detection id know as calibrating .

Then it focus on the paper to read the text. if no line is found it gives the voice message saying that no line has been detcted. If the new line is found is 
found means then it gives the voice message saying that new line is found then it starts reading that line. When the reader reaches end of the line then it give the voice message saying that it is the end of the line. And it returns back to the line not found state.this is how the finger reader works while it is used.

\section{Advantages and disadvantages 6.1 Advantages}

The main advantage of this is to detect the text and gives the voice output for visually impaired.

It helps visually impaired people (VI) to read the text in a sequence order if it misses any line the device vibrates and gives the voice output.

\subsection{Disadvantage}

Finger reader just reads the text and does not specify whether it is headline are sub head line.

The camera does not auto-focus it takes time to focus on the text.

\section{Conclusion}

It is mainly required for the visually impaired (VI) people. By making use of it visually impaired people can easily read the text without anybody's help.
They can also use this to read the text written on the tablet, books, board etc. just by wearing this device visually impaired people can perform text reading operation as a common peoples read the books.

\section{References}

[1] Merrill, D. and Maes, P. Augmenting looking, pointing and reaching gestures to enhance the searching and browsing of physical objects. In Proc. Pervasive'07, (2007) 1-18.

[2] Kane, S. K., Frey, B., and Wobbrock, J. O. Access lens: a gesture-based screen reader for real-world documents. In Proc. of CHI, ACM (2013), 347-350.

[3] Linvill, J. G., and Bliss, J. C. A direct translation readingaid for the blind. Proc. of the IEEE 54, 1 (1966), 40-51.

[4] Chi, L. Y., Ryskamp, R. A., Gomez, L. R. P., Ho, H. And Brin, S. seeing with your hand. Google Patents, 2011.

[5] Smith, R. An overview of the tesseract OCR engine. In Proc. of ICDAR, vol. 2, IEEE (2007), 629-633. 